nature structural \& molecular biology

\title{
Bringing it together
}

\section{An in-depth look at membrane fusion-a process essential for communication within and between cells- is presented in this issue of Nature Structural \& Molecular Biology.}

$\mathrm{W}$

hat do the release of hormones into the bloodstream, the release of digestive enzymes into the gastrointestinal tract, the trafficking and recycling of $G$ protein-coupled receptors to the plasma membrane, and interactions between lysosomes and endosomes all have in common? All of these processes require the fusion of membranes, a regulated process that occurs when two closely apposed membrane compartments are brought together to be joined so that their contents can be mixed. Membrane fusion occurs during the normal life cycle of every cell and is of crucial importance for neurotransmission. It is also the mechanism by which certain viruses enter the cytoplasm of their target host cell.

In this issue of Nature Structural \& Molecular Biology, we present a special Focus on membrane fusion (http://www.nature.com/nsmb/ focus/fusion/index.html), highlighting the main forms of biological membrane fusion and our current understanding of these complex processes, brought together in an Essay and five Reviews. In an accompanying web component, we present an annotated selection of classical papers in the field as well as related work recently published within the pages of the Nature journals.

Much of what is known about intracellular membrane fusion has come from three major approaches: genetic studies in budding yeast, the study of the tightly regulated synaptic fusion machinery and identification of its core components, and lipid-mixing assays aimed at recapitulating the fusion reaction in vitro. These studies have led to the conclusion that fusion is carried out by a largely conserved mechanism performed by the SNARE proteins and associated regulatory factors and effector proteins. Wickner and Schekman (page 658) introduce us to the concept of membrane fusion and provide an overview of current understanding in the field. They also discuss cell-cell fusion and mitochondrial fusion events, two less-well-understood processes that each use a unique repertoire of proteins and probably proceed via mechanisms distinct from intracellular membrane fusion.

Synaptic vesicle fusion is a specialized form of fusion required for neurotransmitter release. This highly controlled event is essential for rapid, precisely timed communication between neurons and can also shape long-term information processing in the brain. The fusion event occurs in response to the influx of $\mathrm{Ca}^{2+}$ into the presynaptic cell, with the synaptic vesicle primed to fuse with the plasma membrane in response to the $\mathrm{Ca}^{2+}$ trigger. In addition to the core fusion machinery, specialized proteins essential to this system have been identified, and recent results have improved our understanding of their role in neurotransmitter release, as discussed by Rizo and Rosenmund (page 665). Despite these advances, it is important to realize that much remains to be determined regarding the complex interplay between these components in regulating the fusion event.

Whereas biological fusion reactions have protein catalysts, studies on protein-free systems have provided a large degree of insight into the mechanical and energetic requirements needed to bring two membranes together. Chernomordik and Kozlov (page 675) present the pros and cons of membrane fusion models based on macroscopic membrane modeling using a classical physics approach and computer-generated molecular dynamics simulations. They also discuss the nature of the pore that forms once the membranes fuse, a topic of intense debate that is also examined in detail by Jackson and Chapman (page 684) in the context of synaptic vesicle fusion.

Enveloped viruses use membrane fusion as a means to enter and infect host cells. Although the protein machinery used to initiate fusion is quite different from SNAREs, extensive structural and functional analysis on viral fusion proteins, as described by Harrison (page 690), provided the first insight into the basic function of the fusion machinery. Namely, in response to a catalyst, the fusion proteins undergo dramatic conformational rearrangements that serve to bring the two membranes together. Because they mediate the point of entry, viral fusion proteins - particularly those of human immunodeficiency virus-1 (HIV-1) and severe acute respiratory syndrome (SARS) - have been the focus of much study and are the targets of ongoing antiviral development programs.

We gratefully acknowledge the authors of these Focus pieces, the reviewers who helped guide them and the advisors who aided in the development of the content for this Focus. We hope that this Focus will bring you up to speed on the latest developments in the field and the important unanswered questions. Our Focus begins with an Essay by Jahn (page 655), who offers a brief history of how this field developed, presenting what he feels are influential papers that have shaped how we think about membrane fusion. 